\title{
Towards Interactive Verification of Programmable Logic Controllers using Modal Kleene Algebra and KIV
}

Roland Glück, Florian Benedikt Krebs

ST-BT

Braga, September 28, 2015 
1. Introduction

2. PLC Crash Course

3. Modal Kleene Algebra and Linear Temporal Logic

4. Function Block Diagrams in Modal Kleene Algebra

5. Case Study: Mutual Exclusion

6. Conclusion and Outlook 

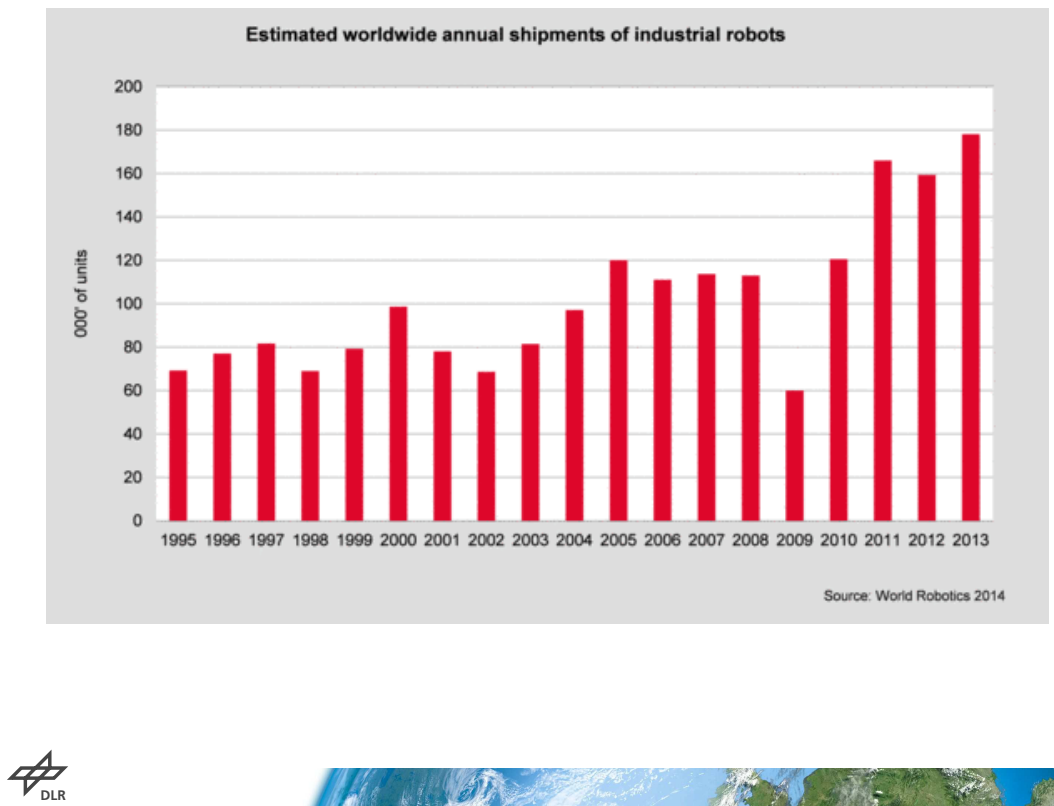

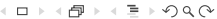


robots are:

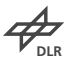

(口) 向, 三

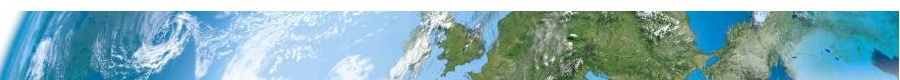


robots are:

- cost saving

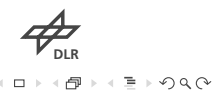


robots are:

- cost saving

- reliable

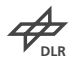

(口) 可, 三 \аल 
robots are:

- cost saving

- reliable

- strong

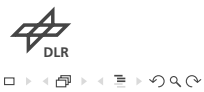


robots are:

- cost saving

- reliable

- strong

- very strong

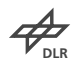

ㅁ. 
robots are:

- cost saving

- reliable

- strong

- very strong

- insensitive

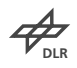

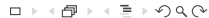


robots are:

- cost saving

- reliable

- strong

- very strong

- insensitive

- dangerous

$\Rightarrow$ careful control is indispensable

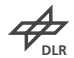

ㅁ. 句 $\equiv$, 


\section{PLC - Purpose and Function}

- Programmable Logic Controllers (PLCs) used for controlling various plants

- robots, pumps, valves, mechanical and automated devices, ...

- PLC works in cyclic way (1 - 150 ms):

- reads input channels (sensors, switches, internal variables)

- computes new values

writes new values to associated output channels/registers (actuators, internal variables)

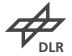

ㅁ. 可 


\section{Data Types and Safety}

- possible data types: bool, int, float, date, ...

- with usual operations (numerical, comparision, ...)

- special part for safety critical operations with reduced instruction set

- from now on only Boolean data and operations 


\section{Programming Languages}

Programming done via:

- Instruction List (IL): assembly-like

- Ladder Diagram (LD): similar to circuit diagrams

- Sequential Function Chart (SFC): inspired by state diagrams

- Structured Text (ST): resembles $C$ syntax

- Function Block Diagram (FBD): see next

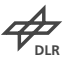

ㅁ. 可 


\section{AND, OR and Negation in FBD}

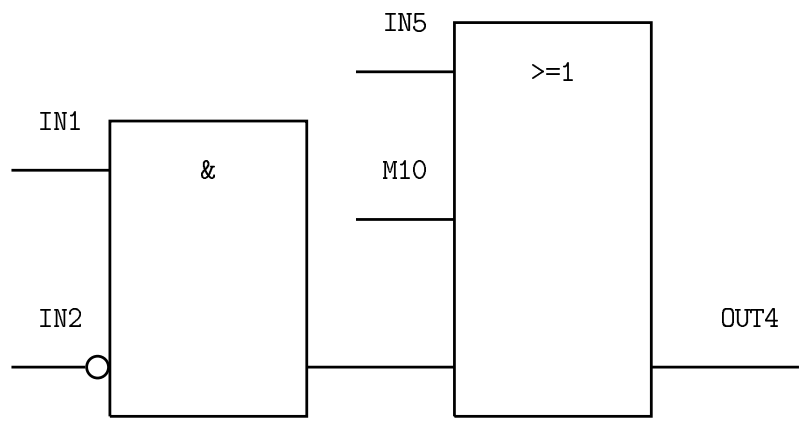

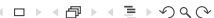




\section{AND, OR and Negation in FBD}

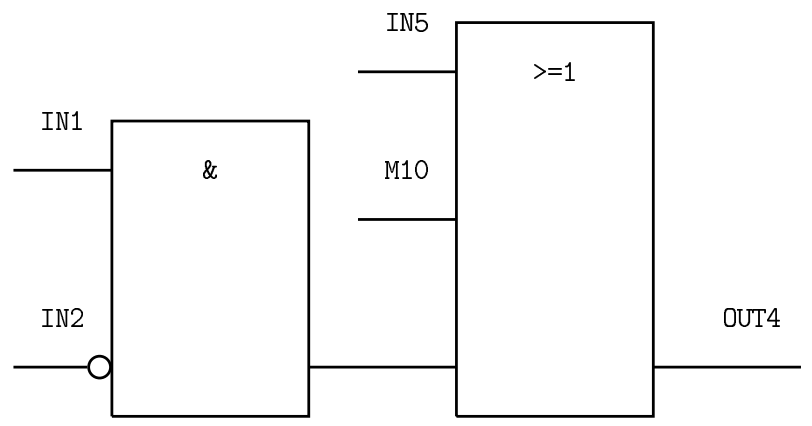

$$
\text { OUT4 } \equiv(\text { IN1 } \wedge \neg \text { IN2 }) \vee \text { IN5 } \vee \text { M10 }
$$




\section{Flip-Flops (Purpose and Function)}

- Flip-Flops show dynamic behavior

- two inputs and one output

- TRUE-signal on set input sets output persistently to TRUE

- TRUE-signal on reset input resets output persistently to FALSE

- (until next signal on set/reset input)

- set/reset dominant depending on winner at set/reset conflict

- storing/clearing depending on input signals 


\section{Flip-Flops (Truth Table)}

\begin{tabular}{|c|c|c|}
\hline $\mathrm{S}_{n}$ & $\mathrm{R}_{n}$ & $\mathrm{Q}_{n+1}$ \\
\hline TRUE & FALSE & TRUE \\
\hline FALSE & TRUE & FALSE \\
\hline FALSE & FALSE & $\mathrm{Q}_{n}$ \\
\hline TRUE & TRUE & TRUE (set dominant) \\
\hline TRUE & TRUE & FALSE (reset dominant) \\
\hline
\end{tabular}




\section{Flip-Flops (FBD)}
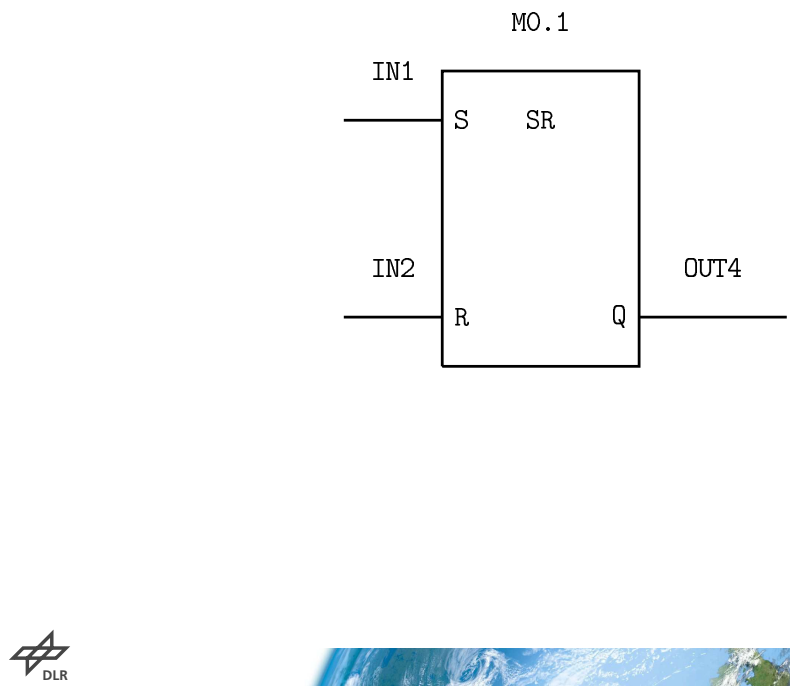

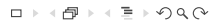




\section{Kleene Algebra}

\section{Definition}

A Kleene algebra is a structure $(M,+, 0, \cdot, 1, *)$ where $(M,+, 0, \cdot, 1)$ is an idempotent semiring and ${ }^{*}: M \rightarrow M$ has the following properties:

$$
\begin{array}{ll}
1+x x^{*} \leq x^{*} & x+y z \leq z \Rightarrow y^{*} x \leq z \\
1+x^{*} x \leq x^{*} & x+y z \leq y \Rightarrow x z^{*} \leq y
\end{array}
$$

- + models choice, · composition, * iteration

- natural order defined by $x \leq y \Leftrightarrow \Leftrightarrow_{d f} x+y=y$

- examples: formal languages, relations, ... 


\section{Tests}

given an idempotent semiring $S=(M,+, 0, \cdot, 1)$ subsets of $M$ can be modeled by tests:

\section{Definition}

Given an idempotent semiring $S=(M,+, 0, \cdot 1)$ an element $p \in M$ is called a test if an element $\neg p$ (the complement of $p$ ) exists with the properties $p+\neg p=1$ and $p \cdot \neg p=0$ $=\neg p \cdot p$.

- set of tests denoted by test $(S)$

- in relational context: subsets of identity 


\section{Boxes and Diamonds}

(pre)image or (pre I post)condition modeled by diamond/box operators:

\section{Definition}

A modal semiring is a structure $S=(M,+, 0, \cdot, 1,|\cdot\rangle,\langle\cdot|)$ where $S^{\prime}=(M,+, 0, \cdot, 1)$ is an idempotent semiring and $|\cdot\rangle$ and $\langle\cdot|$ are functions of the type $M \rightarrow\left(\operatorname{test}\left(S^{\prime}\right) \rightarrow \operatorname{test}\left(S^{\prime}\right)\right)$ with the properties $|x\rangle p \leq q \Leftrightarrow \neg q x p \leq 0 \Leftrightarrow\langle x|p \leq \neg q| x y\rangle p=,|x\rangle|y\rangle p$ and $\langle x y| p=$ $\langle y|\langle x| p$ for all $x \in M$ and $p, q \in S^{\prime}$.

- $\mid$ a)p : transition into $p$ is possible

- $\mid a] p={ }_{d f} \neg|a\rangle \neg p$ : transition into $p$ is inevitable 


\section{Modal Kleene Algebra}

putting all together:

\section{Definition}

A modal Kleene algebra (MKA for short) is a structure $\left(M,+, 0, \cdot, 1,|\cdot\rangle,\langle\cdot|,{ }^{*}\right)$ where $(M,+, 0, \cdot, 1,|\cdot\rangle,\langle\cdot|)$ is a modal semiring and $(M,+, 0, \cdot, 1, *)$ is a Kleene algebra. 


\section{Modal Kleene Algebra and Linear Temporal Logic}

work by Möller, Höfner and Struth (2006):

- model transition system by a general MKA element a

- transforming sets of traces into sets of successors

- left total function modeled by $|a\rangle p=|a| p$ for all tests $p$

- formulae in linear temporal logic (LTL) correspond to expressions in MKA

- LTL formula is valid iff corresponding MKA expression evaluates to 1

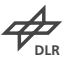

ㅁ. 可 $\equiv \curvearrowleft Q \curvearrowright$ 


\section{Explicit Correspondence}

$$
\begin{aligned}
& \llbracket \perp \rrbracket \quad=\quad 0 \\
& \llbracket \neg \psi \rrbracket \quad=\quad \neg \llbracket \psi \rrbracket \\
& \llbracket \psi_{1} \wedge \psi_{2} \rrbracket=\llbracket \psi_{1} \rrbracket \cdot \llbracket \psi_{2} \rrbracket \\
& \llbracket \psi_{1} \vee \psi_{2} \rrbracket=\llbracket \psi_{1} \rrbracket+\llbracket \psi_{2} \rrbracket \\
& \llbracket \psi_{1} \rightarrow \psi_{2} \rrbracket=\llbracket \psi_{1} \rrbracket \rightarrow \llbracket \psi_{2} \rrbracket \quad\left(p \rightarrow q={ }_{d f} \neg p+q\right) \\
& \left.\llbracket \square \psi \rrbracket \quad=\quad \llbracket \mid a^{*}\right] \psi \rrbracket \\
& \llbracket \diamond \psi \rrbracket \quad=\quad \llbracket\left|a^{*}\right\rangle \psi \rrbracket \\
& \llbracket \circ \psi \rrbracket \quad=\quad \llbracket|a\rangle \psi \rrbracket \\
& \llbracket \psi_{1} \cup \psi_{2} \rrbracket=\left|\left(\llbracket \psi_{1} \rrbracket \cdot a\right)^{*}\right\rangle \llbracket \psi_{2} \rrbracket
\end{aligned}
$$




\section{wwww.dlr.de Slide 17 of $26>$ PLC Verification with MKA and KIV > Roland Glück, Florian Benedikt Krebs · Braga, September 28,2015}

\section{Variables and Overall Behavior}

FBDs in MKA:

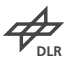

(口) 向, 三 
FBDs in MKA:

- inputs/outputs/internal variables correspond to tests

- for every signal/variable $\mathrm{p}$ introduce two tests p_0 and p_1

- indicating a value of FALSE and TRUE, resp.

- clearly $\neg$ p_0 = p_1 and $\neg$ p_1 = p_0

- characterize behavior of elementary gates (OR, AND, Flip-Flops, ...)

- elementary gates do not change noninvolved signals/variables

- remember left total functionality

- write overall behavior a as product of elementary gates

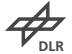




\section{Elementary Gates}

- AND-gate ANDk with inputs in1, in2 ..., inn :

- in1_1 - in2_1 $\cdots$. inn_1 $\leq$ andk $\rangle$ andk_1

- in1_0 + in2_0 + $\cdots+$ inn_0 $\leq \mid$ andk $\rangle$ andk_0.

- OR-gate ORk with inputs in1, in2 ..., inn :

- in1_1 + in2_1 + - + inn_1 $\leq$ ork $>$ ork_1

- in1_0.in2_0 ..... inn_0 $\leq$ ork $>$ ork_0.

- negation of sk : switch sk_1 and sk_0

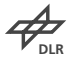




\section{Flip-Flops}

- set dominant flip-flop RSk with set input $\mathbf{s}$, reset input $r$, output $q$ and internal marker $\mathrm{m}$ :

- $s_{-} 1+m_{-} 1 \cdot r \_0 \leq|r s k\rangle q \_1$

- $\mathrm{s}_{-} 1+\mathrm{m} \_1 \cdot \mathrm{r} \_0 \leq|\mathrm{rsk}\rangle \mathrm{m} \_1$

- s_0 $r_{-} 1+m_{-} 0 \cdot s_{-} 0 \leq|r s k\rangle q \_0$

- $s_{-} 0 \cdot r_{-} 1+m_{-} 0 \cdot s_{-} 0 \leq|r s k\rangle m_{-} 0$

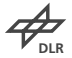




\section{Example Construction (not Complete!)}

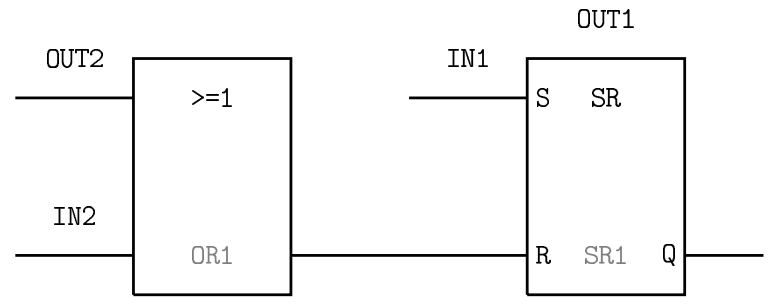

$$
\begin{aligned}
& \text { out2_1 + in2_1 } \leq \text { or1 } 1 \text { or1_1 } \\
& \text { out2_0 - in2_0 } \leq \text { or } 1\rangle \text { or1_0 } \\
& \text { in1_0 } \leq \text { or } 1\rangle \text { in1_0 } \\
& \text { in1_1 } \leq \text { or } 1\rangle \text { in1_1 } \\
& \text { or } 1\rangle p=\mid \text { or } 1] p
\end{aligned}
$$
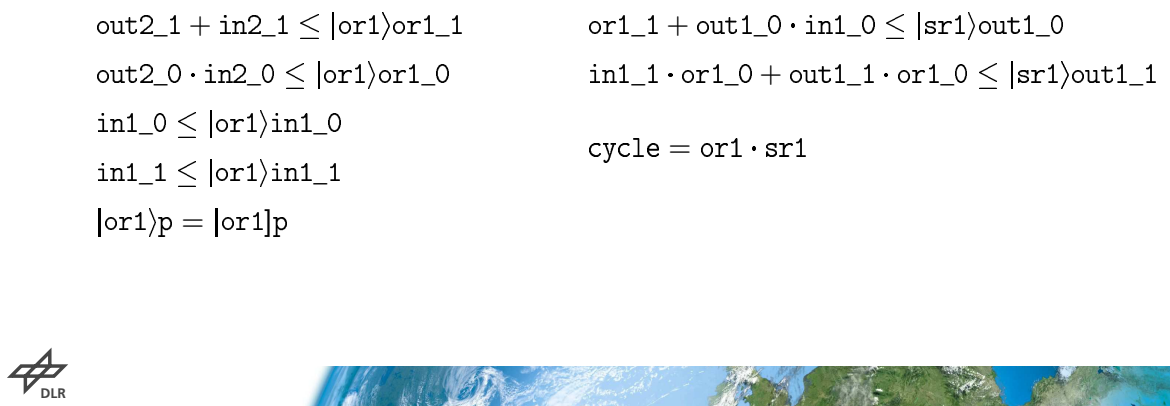


\section{Mutual Exclusion}
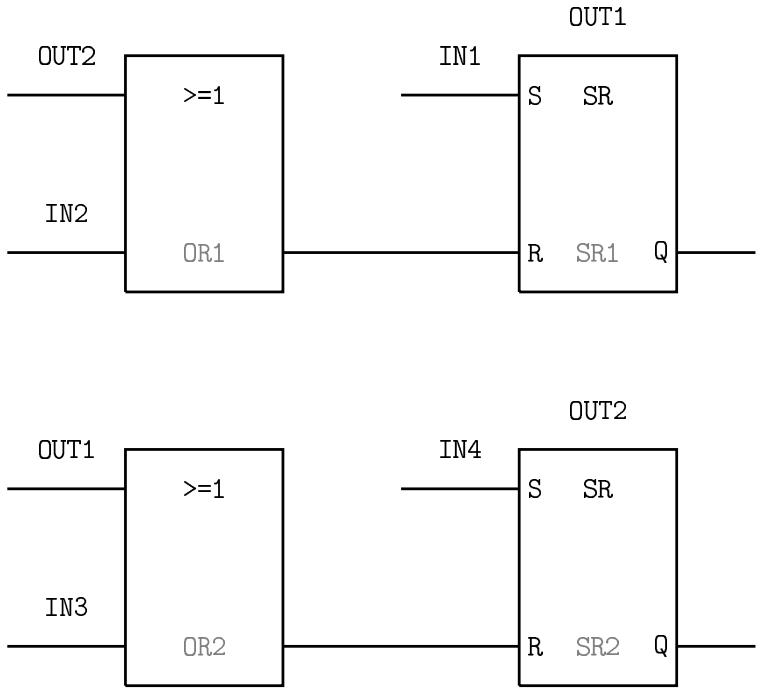

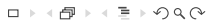




\section{Behavior and Desired Properties}

- behavior given by cycle $=$ or $1 \cdot \operatorname{sr} 1 \cdot \operatorname{or} 2 \cdot \operatorname{sr} 2$

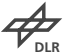

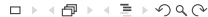




\section{Behavior and Desired Properties}

- behavior given by cycle $=$ or $1 \cdot \operatorname{sr} 1 \cdot$ or $2 \cdot \operatorname{sr} 2$

- desired properties in LTL:

- out1_0 - out2_0 $\rightarrow \square($ out1_1 $\rightarrow$ out2_0)

- out1_0 - out2_0 $\rightarrow \square($ out2_1 $\rightarrow$ out1_0)

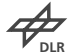

ㅁ. 


\section{Behavior and Desired Properties}

- behavior given by cycle $=$ or $1 \cdot \operatorname{sr} 1 \cdot$ or $2 \cdot \operatorname{sr} 2$

- desired properties in LTL:

- out1_0 - out2_0 $\rightarrow \square($ out1_1 $\rightarrow$ out2_0)

- out1_0 - out2_0 $\rightarrow \square($ out2_1 $\rightarrow$ out1_0)

- in MKA (recall $\left.p \rightarrow q={ }_{d f} \neg p+q\right)$ :

- out1_0 - out2_0 $\rightarrow \mid$ cycle*](out1_1 $\rightarrow$ out2_0) $=1$

- out1_0 - out2_0 $\rightarrow \mid$ cycle*](out2_1 $\rightarrow$ out1_0 $)=1$

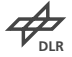




\section{Proof Sketch}

to show: out1_0 - out2_0 $\rightarrow \mid c y c l e *]($ out1_1 $\rightarrow$ out2_0 $)=1$

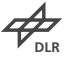

ㅁ. 可, $\equiv$, $9 \curvearrowright$ 


\section{Proof Sketch}

to show: out1_0 out2_0 $\rightarrow \mid$ cycle* $]($ out1_1 $\rightarrow$ out2_0 $)=1$

proof sketch:

- first: out1_0 out2_0 + out1_0 out2_1 + out1_1 out2_0 is an invariant of cycle

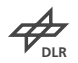

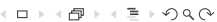




\section{Proof Sketch}

to show: out1_0 out2_0 $\rightarrow \mid$ cycle $\left.e^{*}\right]($ out1_1 $\rightarrow$ out2_0 $)=1$

proof sketch:

- first: out1_0 out2_0 + out1_0 out2_1 + out1_1 out2_0 is an invariant of cycle

- MKA: out1_0 out2_0 + out1_0 out2_1 + out1_1 out2_0 is an invariant of cycle*

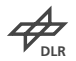

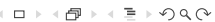




\section{Proof Sketch}

to show: out1_0 out2_0 $\rightarrow \mid$ cycle* $]($ out1_1 $\rightarrow$ out2_0 $)=1$

proof sketch:

- first: out1_0 out2_0 + out1_0 out2_1 + out1_1 out2_0 is an invariant of cycle

- MKA: out1_0 out2_0 + out1_0 out2_1 + out1_1 out2_0 is an invariant of cycle*

- MKA: $p \leq q \wedge q x \neg q=0 \wedge q \leq r \Rightarrow p \rightarrow|x| r=1$

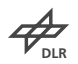

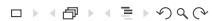




\section{Proof Sketch}

to show: out1_0 out2_0 $\rightarrow \mid$ cycle $\left.e^{*}\right]($ out1_1 $\rightarrow$ out2_0 $)=1$

proof sketch:

- first: out1_0 out2_0 + out1_0 out2_1 + out1_1 out2_0 is an invariant of cycle

- MKA: out1_0 out2_0 + out1_0 out2_1 + out1_1 out2_0 is an invariant of cycle*

- MKA: $p \leq q \wedge q x \neg q=0 \wedge q \leq r \Rightarrow p \rightarrow|x| r=1$

- finish:

- out1_0 out2_0 $\leq$ out1_0 out2_0 + out1_0 out2_1 + out1_1 out2_0

- out1_0 out2_0 + out1_0 out2_1 + out1_1 - out2_0 $\leq$ out1_1 $\rightarrow$ out2_0

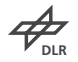




\section{Proof Sketch}

to show: out1_0 • out2_0 $\rightarrow \mid$ cycle*](out1_1 $\rightarrow$ out2_0 $)=1$

proof sketch:

- first: out1_0 out2_0 + out1_0 out2_1 + out1_1 out2_0 is an invariant of cycle

- MKA: out1_0 out2_0 + out1_0 out2_1 + out1_1 out2_o is an invariant of cycle*

- MKA: $p \leq q \wedge q x \neg q=0 \wedge q \leq r \Rightarrow p \rightarrow|x| r=1$

- finish:

- out1_0 out2_0 $\leq$ out1_0 out2_0 + out1_0 out2_1 + out1_1 out2_0

- out1_0 - out2_0 + out1_0 out2_1 + out1_1 out2_0 $\leq$ out1_1 $\rightarrow$ out2_0

- proof done interactively in KIV

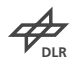

ㅁ. 可 


\section{Conclusion}

We saw:

4

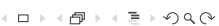




\section{Conclusion}

We saw:

- Programmable Logic Controllers

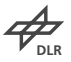

ㅁ. 可, $\equiv$ ac 


\section{Conclusion}

We saw:

- Programmable Logic Controllers

- Modal Kleene Algebra

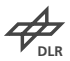

ㅁ. 


\section{Conclusion}

We saw:

- Programmable Logic Controllers

- Modal Kleene Algebra

- Linear Temporal Logic

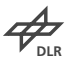




\section{Conclusion}

We saw:

- Programmable Logic Controllers

- Modal Kleene Algebra

- Linear Temporal Logic

- interactive proving with KIV

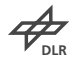




\section{Conclusion}

We saw:

- Programmable Logic Controllers

- Modal Kleene Algebra

- Linear Temporal Logic

- interactive proving with KIV

- and all working together

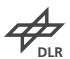

ㅁ 可 $\equiv \curvearrowleft 9 \Omega$ 


\section{Outlook}

We plan:

4

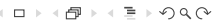

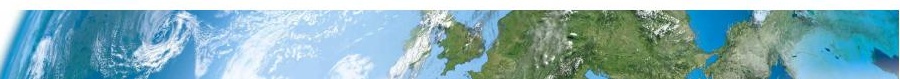




\section{Outlook}

We plan:

- verification of real safety systems

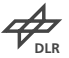

ㅁ 


\section{Outlook}

We plan:

- verification of real safety systems

- typical features:

- 32 - 64 signals from sensors

- plus up to 16 signals from safety doors

- 50 - 100 elementary gates

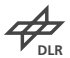




\section{Outlook}

We plan:

- verification of real safety systems

- typical features:

- 32 - 64 signals from sensors

- plus up to 16 signals from safety doors

- 50 - 100 elementary gates

- characterization of other gates in MKA

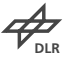




\section{Outlook}

We plan:

- verification of real safety systems

- typical features:

- 32 - 64 signals from sensors

- plus up to 16 signals from safety doors

- 50 - 100 elementary gates

- characterization of other gates in MKA

- embracing numerical operations

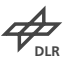

ㅁ. 可 $\equiv \curvearrowleft Q \curvearrowright$ 


\section{Outlook}

We plan:

- verification of real safety systems

- typical features:

- 32 - 64 signals from sensors

- plus up to 16 signals from safety doors

- 50 - 100 elementary gates

- characterization of other gates in MKA

- embracing numerical operations

- timer

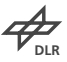

ㅁ. 可 $\equiv \curvearrowleft Q \curvearrowright$ 


\section{Outlook}

We plan:

- verification of real safety systems

- typical features:

- 32 - 64 signals from sensors

- plus up to 16 signals from safety doors

- 50 - 100 elementary gates

- characterization of other gates in MKA

- embracing numerical operations

- timer

- automated construction of input files

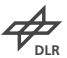

ㅁ. 

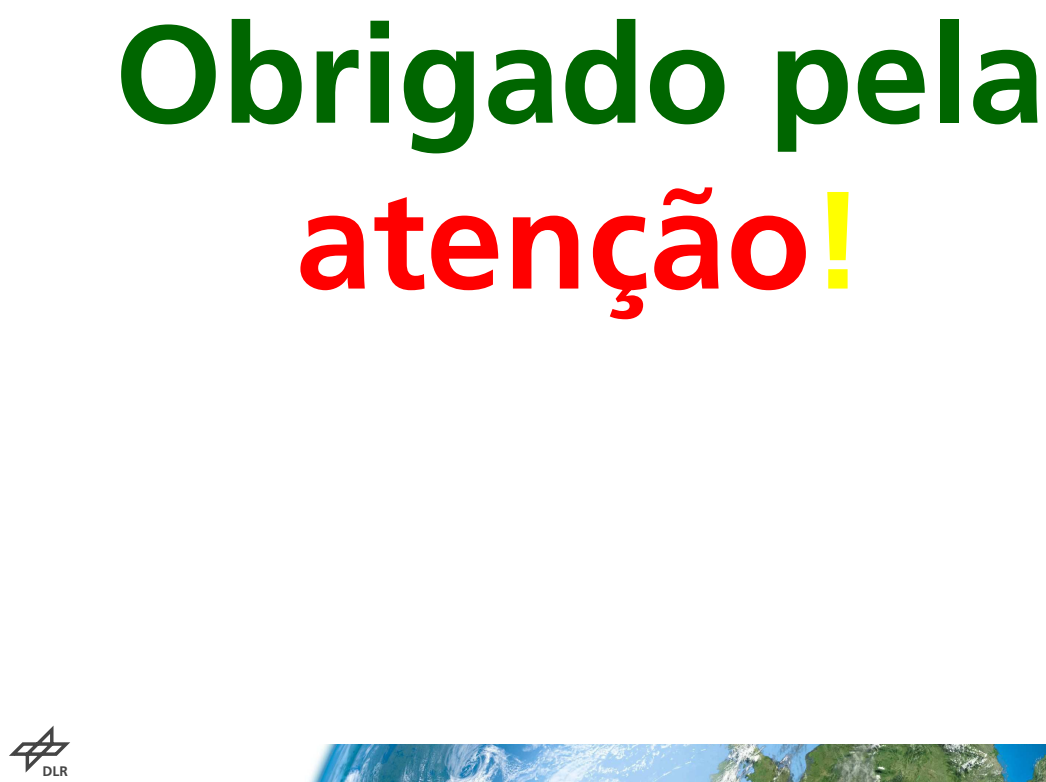

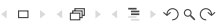



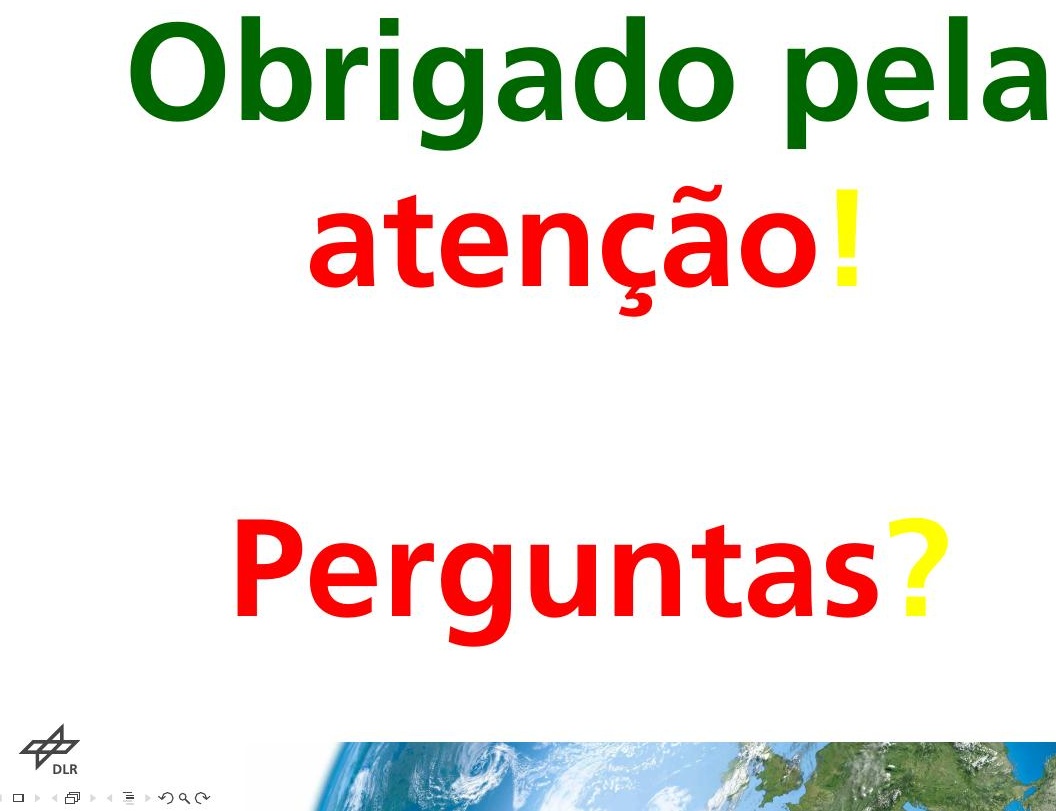